\title{
Effect of Spinal Stimulation on Monosynaptic Reflex by Medium Frequency Current
}

\author{
Nidhi $^{1}$, B., Narkeesh ${ }^{2}$, A. \& Khurana ${ }^{3}$, S. \\ ${ }^{1}$ Post graduate student of Neurological Physiotherapy, Sardar Bhagwan Singh Post Graduate \\ Institute, Dehradun. \\ ${ }^{2}$ Associate Professor, Department of Physiotherapy, Punjabi University, Patiala. \\ ${ }^{3}$ Assistant. Professor, Department of Physiotherapy, SBSPGI, Dehradun
}

\begin{abstract}
Surface spinal stimulation with electrical stimulation plays a major role in the management of various neurological disorders and also reduces the spasticity. There are many previous studies where surface spinal stimulation had been used in various neurological disorders and purposes and mostly in these studies low frequency current are used but still there is no study to explain how surface spinal stimulation by two medium frequency currents influences on monosynaptic reflex. In present study Premodular IFC \& Russian current by surface spinal stimulation has been used at T-12 \& L-1 for 45 minutes to see how it influence on monosynaptic reflex by taking Pre \& post readings. The mean, standard deviations were calculated for all variables. After that ANOVA was applied between pre \& post reading of all variables after medium frequency currents and after that two currents were compared by paired $t$ - value for all the parameters of monosynaptic reflex. It was concluded that spinal stimulation with medium frequency current resulted in a significant effect on monosynaptic reflex as there was significant effect on H-latency and H-amplitude, however there was non significant effect on H/M ratio \& Premodular IFC produced greater effect than the Russian current.
\end{abstract}

Key words: Monosynaptic reflex, Surface Spinal Stimulation, Medium frequency currents

\section{Introduction}

Electrotherapy is the treatment of patients by electrical means; in this electrical forces are applied to the body through which physiological changes occur for therapeutic purposes. Electrical stimulation helps in reducing spasticity in case of SCI, Cerebral palsy and Multiple sclerosis patients along with improvement in bladder function, respiratory function volitional control; active and passive movement and mood state with carry over effects lasting from 30 minutes to 24 hours (Hazelewood et al, 1994). American Physical Therapy Association acknowledges the use of electrotherapy in various fields such as pain management, tone management, treatment of neuromuscular dysfunction, improves range of joint mobility, tissue repair, acute and chronic edema, peripheral blood flow, iontophoresis, Urine and fecal incontinence (Alon et al, 2005).

Surface Spinal Stimulation is delivered by pulses that are generated from amplitude modulated alternating current of a carrier frequency of $2500 \mathrm{~Hz}$, and modulated to deliver beat at a frequency of $20 \mathrm{~Hz}$. For application, an electrode is placed on each side of the spine (5cm apart) over the paravertebral skin at the twelfth thoracic and first lumbar vertebral levels. The self-adhesive electrodes of rectangular shape and size of $5 \times 9 \mathrm{~cm}$ are used. The stimulation amplitude is adjusted for each subject to produce only sensory stimulation and it is 
applied continuously for 45 minutes (Wang et al, 2000).

Surface spinal stimulation through Tens also produces a significant effect on monosynaptic reflex (Arsenault, 1993). In the present study attempt has been made to find physiological reasoning how surface spinal stimulation influences the monosynaptic stretch reflex in 30 normal adolescents and its physiological changes takes place through surface spinal stimulation and how different medium frequency currents produce effects on monosynaptic reflex.

\section{Methods}

Study was performed on 30 female subjects taken from the SBSPGI, Dehradun under the age group of 20-25 years. It was an experimental study which was performed in the Department of Physiotherapy. Study was performed in accordance with the ethical considerations of the institute and the consent of the subjects was taken prior to the study.

Before beginning with the procedure, the subjects were selected on the basis of convenient sampling by applying inclusion criteria and were explained the entire procedure in detail. The inclusion criteria were (i) female subjects of age 20 to 25 years, (ii) with normal BMI between 19 to $25 \mathrm{~kg} / \mathrm{m}^{2}$ and with (iii) height $165-190 \mathrm{~cm}$ are included. The body temperature ranged between $36-$ $37^{\circ} \mathrm{C}$, leg length from $27-49 \mathrm{~cm}$ and absence of any systemic, physical or neurological problem. The Exclusion Criteria was any sensory or motor impairment, space occupying lesion, upper motor neuron or lower motor neuron symptoms, mixed symptom rigidity, flaccidity and spasticity, any neurological and cognitive deficit, any radiculopathy and neuropathy, any recent surgery on back, hip, knee, height below $165 \mathrm{~cm}$ or above $190 \mathrm{~cm}$, leg length less than $27 \mathrm{~cm}$ or more than $49 \mathrm{~cm}$ and subjects with BMI more than 25 or less than 19.

Of all the 30 subjects, H-reflex was recorded maintaining room temperature between $19-24^{\circ} \mathrm{C}$ on non-dominant side in prone lying. Recording and reference electrodes were placed at the soleus muscle and sub maximal stimulation was given at popliteal crease. Surface spinal stimulation with premodular IFC was then applied at $\mathrm{T}_{12} \& \mathrm{~L}_{1}$ level with rectangular shaped electrodes of $4.5 \times 9.5 \mathrm{~cm}$ for 45 minutes in prone lying and again 3 readings of H-reflex were recorded after 5 minutes, 10 minutes \& 15 minutes of treatment. After that washout period of 30 minutes were given to the subject $\&$ again pre reading was taken \& after that again Russian current was applied for 45 minutes \& whole procedure was repeated \& Pre and post stimulation values of latency of H-reflex (ms), H/M ratio and amplitude of $\mathrm{H}$-wave $(\mathrm{mV})$ were taken. The data was collected and analyzed.

\section{Results}

Table 1: Mean and SD of Age, Height \& Weight

\begin{tabular}{ccc}
\hline Demographic & MEAN & SD \\
\hline Age & 21.03 & 1.52 \\
Height & 5.24 & 0.14 \\
Weight & 51.7 & 0.14 \\
\hline
\end{tabular}

Table 1 shows the mean and standard deviation values of age, height and weight of the subjects. The mean and standard deviation of age is $21.03 \pm 1.52$ yrs._The mean height was $5.24 \pm .14 \mathrm{ft}$ and weight was $51.7 \pm .14 \mathrm{~kg}$ and mean BMI was 20.56 \pm 1.78 . Repeated measures ANOVA shows a significant difference in Latency \& amplitude values across the time 
points. A simple contrast results represent statistical significant difference between the pre-value and 5-minutes $(\mathrm{P}<0.01)$, and 10 -minutes $(\mathrm{P}<0.01$, but not in 15-minutes amplitude values $(\mathrm{P}=0.714)$.

Table 2: Mean and SD of H-Latency, H/M and $\mathrm{H}$ amplitude at Pre and Post intervals after Premodular IFC \& comparison of mean value pre $\&$ post values

\begin{tabular}{|c|c|c|c|c|c|}
\hline & & \multirow{2}{*}{ Mean } & \multirow{2}{*}{ SD } & \multirow{2}{*}{$\begin{array}{l}\text { F-value/P-value } \\
\text { (repeated measures ANOVA) }\end{array}$} & \multirow{2}{*}{$\begin{array}{l}\text { Significance } \\
\text { (Compared with pre-value)* }^{*}\end{array}$} \\
\hline & & & & & \\
\hline \multirow[t]{4}{*}{ H-Latency } & Pre-value & 27.53 & 1.22 & \multirow{4}{*}{$\begin{array}{c}\mathrm{F}=\mathbf{1 1 . 4 4 8}(\mathrm{df}=\mathbf{3}, 87) \\
\mathrm{P}=\mathbf{0 . 0 0 0} \\
(\mathrm{P}<\mathbf{0 . 0 0 1})\end{array}$} & \\
\hline & 5-minutes & 28.20 & 1.02 & & $P=0.009$ \\
\hline & 10-minutes & 28.34 & 1.06 & & $P=0.000(P<0.001)$ \\
\hline & 15-minutes & 28.30 & 0.99 & & $P=0.000(P<0.001)$ \\
\hline \multirow[t]{4}{*}{ H-Amp } & Pre-value & 3.19 & 1.19 & \multirow{4}{*}{$\begin{array}{c}\mathrm{F}=27.131(\mathrm{df}=3,87) \\
P=0.000 \\
(P<0.001)\end{array}$} & \\
\hline & 5-minutes & 4.02 & 1.04 & & $P=0.000(P<0.001)$ \\
\hline & 10-minutes & 4.79 & 1.08 & & $P=0.000(P<0.001)$ \\
\hline & 15-minutes & 3.76 & 1.22 & & $P=0.039$ \\
\hline \multirow[t]{3}{*}{ H/M Ratio } & 5-minutes & 0.139 & 0.09 & $\begin{array}{l}F=.710(d f=3,87) \\
P=0.000(P<0.001)\end{array}$ & \\
\hline & 10-minutes & 0.174 & 0.146 & & $P=0.548$ \\
\hline & 15minutes & 0.166 & 0.097 & & $P=0.548(P>.005)$ \\
\hline
\end{tabular}

Table 3: Mean and SD of H-Latency, $\mathrm{H} / \mathrm{M}$ and $\mathrm{H}$ amplitude at Pre and Post intervals after Russian current \& comparison of mean value pre $\&$ post values

\begin{tabular}{|c|c|c|c|c|c|}
\hline & & Mean & SD & $\begin{array}{l}\text { F-value/P-value } \\
\text { (repeated measures ANOVA) }\end{array}$ & $\begin{array}{l}\text { Significance } \\
\text { (Compared with pre-value) }\end{array}$ \\
\hline \multirow[t]{4}{*}{ H-Latency } & Pre-value & 27.53 & 1.22 & & \\
\hline & 5-minutes & 28.20 & 1.02 & \multirow{3}{*}{$\begin{array}{l}F=11.448(\mathrm{df}=3,87) \\
P=0.000 \\
(P<0.001)\end{array}$} & $P=0.009$ \\
\hline & 10-minutes & 28.34 & 1.06 & & $P=0.000(P<0.001)$ \\
\hline & 15-minutes & 28.30 & 0.99 & & $P=0.000(P<0.001)$ \\
\hline \multirow[t]{4}{*}{ H-Amp } & Pre-value & 3.19 & 1.19 & & \\
\hline & 5-minutes & 4.02 & 1.04 & \multirow{3}{*}{$\begin{array}{l}F=27.131(d f=3,87) \\
P=0.000 \\
(P<0.001)\end{array}$} & $P=0.000(P<0.001)$ \\
\hline & 10-minutes & 4.79 & 1.08 & & $P=0.000(P<0.001)$ \\
\hline & 15-minutes & 3.76 & 1.22 & & $P=0.039$ \\
\hline \multirow[t]{3}{*}{ H/M ratio } & 5-minuts & 0.125 & 0.082 & $\begin{array}{l}F=.681 \\
P=0.565(P<0.01\end{array}$ & \\
\hline & 10-minutes & 0.14 & 0.13 & & $P=0.565$ \\
\hline & 15-minutes & 0.14 & 0.093 & & $P=0.565(P<.005)$ \\
\hline
\end{tabular}

The mean value depicts an increase in amplitude value from baseline upto 10 minutes and then starts decreasing where as latency increases from baseline to 15 . To compare the Pre H/M ratio with other time points ANOVAs was applied. Only 5-minutes value was found to be significant with pre value. While post values show a non significant result of $\mathrm{H} / \mathrm{M}$ ratio. 
Table 4: Comparison of both premodular IFC and Russian Current

\begin{tabular}{ccccccc} 
& & \multicolumn{2}{c}{ IFS } & \multicolumn{2}{c}{ Russian } & P-value \\
\hline & & Mean & SD & Mean & SD & (Paired t-test) \\
\hline H-Latency & Pre-value & 27.53 & 1.22 & 27.44 & 1.24 & 0.228 \\
& 5-minutes & 28.20 & 1.02 & 27.90 & 1.33 & 0.195 \\
& 10-minutes & 28.34 & 1.06 & 28.00 & 1.37 & 0.082 \\
& 15-minutes & 28.30 & 0.99 & 28.08 & 1.59 & 0.355 \\
H-Amp & Pre-value & 3.19 & 1.19 & 3.16 & 1.18 & 0.661 \\
\hline & 5-minutes & 4.02 & 1.04 & 3.69 & 1.35 & 0.074 \\
& 10-minutes & 4.79 & 1.08 & 4.05 & 1.23 & 0.008 \\
& 15-minutes & 3.76 & 1.22 & 3.00 & 1.11 & 0.002 \\
\hline \multirow{2}{*}{ H/M Ratio } & Pre-value & 0.139 & 0.09 & 0.125 & 0.082 & 0.114 \\
& 5minutes & 0.174 & 0.146 & 0.14 & 0.13 & 0.416 \\
& 10-minutes & 0.166 & 0.097 & 0.14 & 0.093 & 0.22 \\
& 15minute & 0.137 & 0.141 & 0.12 & 0.1005 & 0.488 \\
\hline
\end{tabular}

The table 4 shows comparison of $\mathrm{H}$ amplitude between the Premodular IFC and Russian current. After spinal stimulation the pre readings were found to be ( $\mathrm{t}$ - value $0.443, \mathrm{p}$ value $<0.05)$, after 5 minutes (t-value -1.734 , $\mathrm{p}$ - value $<0.05$ ), after 10 minutes ( $\mathrm{t}-$ value -2.869 , $\mathrm{p}$ value $<0.05)$ and after 15 minutes ( $\mathrm{t}-$ value 3.319 , p value < 0.05) were found to be non significant for pre reading and for 5 minutes and significant for 10 minutes and 15 minutes of treatment. While $\mathrm{H}$ latency \& $\mathrm{H} / \mathrm{M}$ ratio show nonsignificant results.

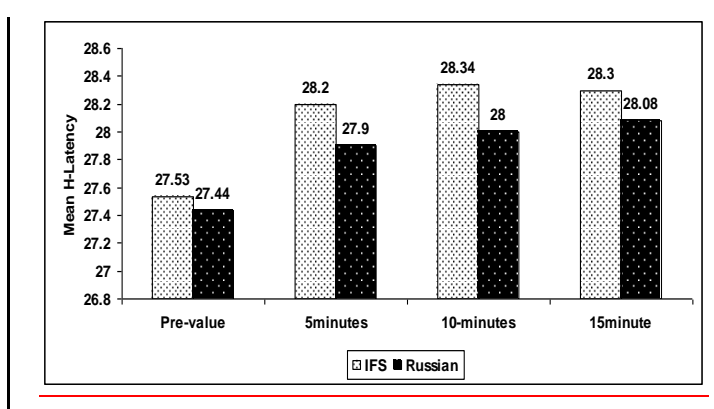

Figure 1: Comparison of H-latency of both premodular IFC \& Russian current

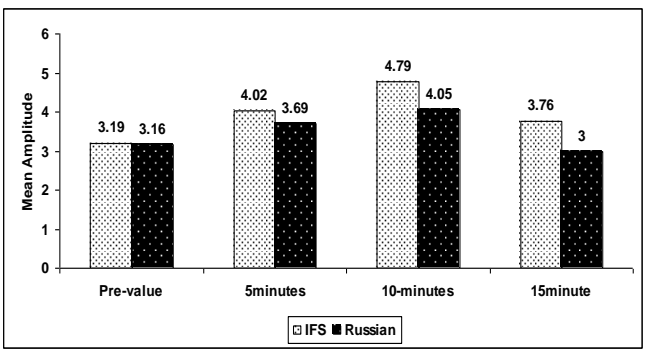

Figure 2: Comparison of Mean $\mathrm{H}$-amplitude of both premodular IFC \& Russian current

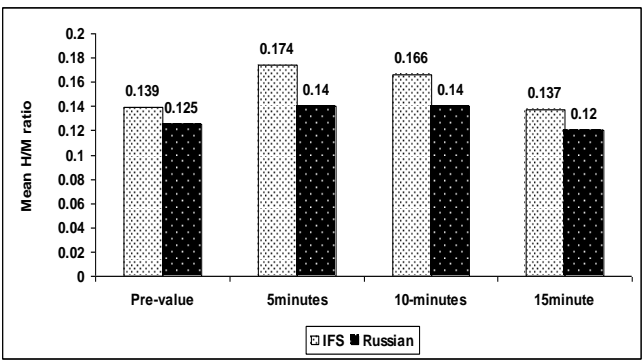

Figure 3: Comparison of Mean H/M Ratio of both premodular IFC \& Russian current

\section{Discussion}

Electrical stimulation is well known technique that uses electrical currents for the management of various neurological disorders. Electrical stimulation is effective in the treatment of SCI patients as it reduces spasticity in them (Lojze 
Vodovnik, 1984). Stimulation of spastic muscle itself is based on recurrent inhibition which is caused by Renshaw cell, which has a negative feedback loop to the motorneurons (Rothwell et al, 1994). Surface spinal stimulation has pronounced effects in H-reflex, which is an electrically induced reflex analogous to mechanically induced spinal stretch reflex; its arc is similar to spinal stretch reflex except that it bypasses the muscle spindle, and therefore it is a valuable tool for assessing mono synaptic reflex activity in spinal cord. Thus, it is a reliable tool for the assessment of muscle tone through the excitability of alpha motor neuron (Palmieri et al, 2004). In the present study, to find the effect of surface spinal stimulation in H-reflex, only female subjects were taken because the significant role of sex in H-reflex has already been elicited (Huang et al, 2009). H-reflex has also shown modifications due to variations in age and leg length (Robinson et al, 1988). So in the present study, considering the age factor, subjects of age group 20-25 years and leg length in present study were controlled between 27 and $49 \mathrm{~cm}$. H - reflex latency shows increase with cooling and decrease with warming (Riccardo et al, 2001). So, in the present study room temperature was monitored from $19-24^{\circ} \mathrm{C}$. As Body Mass Index has also shown to influence the conduction changes (Buschbacher, 1998), the subjects with normal BMI (19-25 $\mathrm{Kg} / \mathrm{m}^{2}$ ) were included in the study._ In the present study, surface spinal stimulation of carrier frequency of $2500 \mathrm{~Hz}$ and beat frequency $20 \mathrm{~Hz}$ was given through the surface electrodes of size $4.5 \times 9 \mathrm{~cm}$, which were placed $5 \mathrm{~cm}$ apart on each side of spine, as explained by Wang et al (2000).

The results revealed that there are changes in the nerve excitability, $\mathrm{H}$ amplitude and H-Latency and there is significant influence of Surface Spinal Stimulation on monosynaptic reflex. There is significant increase of H-latency and $\mathrm{H}$ amplitude; however there is elongation of $\mathrm{H} / \mathrm{M}$ ratio but not upto significant level. The results are in accordance with study done by Arsenault et al (1993) who also found a gradual increase of H-reflex amplitude upto $40 \%$ after 20 minutes regardless of whether the TENS is applied on L5 or S2. They further reported that increase in $\mathrm{H}$ response value was accompanied by a shift of spectral content towards low frequencies, which occurred with a cooling of skin overlying the soleus muscle. The results of the study are also supported by the study done by Alireza (2009). They however used tripolar TENS with different intensities (1.5 ST and 1.25 ST) on T-11 vertebra which showed peak to peak increase in amplitude as slow motor neurons were inhibited and fast motor neurons were facilitated.

\section{Conclusion}

Results of the present study suggests that spinal stimulation when done with medium frequency currents i.e Premodular IFC $(3000 \mathrm{~Hz})$ and Russian current $(2500 \mathrm{~Hz})$ produces a significant change in H-Latency and H-amplitude and it was also found out that Premodular IFC $(3000 \mathrm{~Hz})$ produces more effect in various parameters of $\mathrm{H}$-reflex such as $\mathrm{H}$ Latency and $\mathrm{H}$-amplitude as compared to Russian current.

\section{References}

Alon $\mathrm{G}$ et al. Electrotherapeutic Terminology in Physical Therapy 2005. Section on Clinical Electrophysiology. Alexandria, VA: American Physical Therapy Association. 
Alireza, S., Firoozabadi, S.M.P., Torkaman, G., Fathollahi, Y. 2009. The effect of vertebral column tripolar electrical stimulation with various intensities on soleus and gastrocnemius H-reflex and $\mathrm{Mh}$ Wave recruitment curve", Physiology and Pharmacology, 13(2): 229-243.

Arsenault, B., Belanger, A.Y., Durand, M.J., De Serres, S.J., Fortin, L., Kemp, F. 1993. Effect of TENS and topical anesthesia on soleus Hreflex and the concomitant influence of skin/ muscle temperature, Arch. Phys. Med. Rehab. 74(1): 48-53.

Buschbacher, R. 1999. Normal range for H-reflex recording from the calf muscle S., $\underline{A m}$. J. Phys. Med. Rehab., 77: 6.

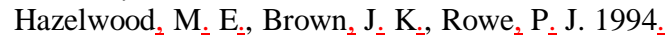
The use of therapeutic electrical stimulation in the treatment of hemiplegic cerebral palsy. Dev. Med. Chil. Neurol.36:-661-673.

Huang, C.R., Chang, W.N., Chang, H.W., Tsai, N.W., and Lu, C. 2009. Effects of Age, Gender, Height and Weight on late responses and nerve conduction study parameters", Acta Neurol Taiwan, 18: 242-249.

Palmieri, R.M., Ingersoll, C.D. and Hoffman, M.A. 2004. The Hofmann Reflex: Methodologic considerations and applications for use in sports medicine and athletic training research, J. Athl. Train., 39(3): 268-77.

Riccardo, M., Giovani, B.S., Aido, M., Vitaliano, F.M., Lucio, P. 2001. Recruitment curve of the Soleus H-reflex in chronic back pain and lumbosacral radiculopathy, BMC Muscle Skeletal Disorders, 4: 11

Robinson, C., Kett, N., Bolam, J. 1988. Spasticity in Spinal Cord injured patients: 2. Initial measures and long term effects of surface electrical stimulation. Arch. Phys. Med. Rehabil., 69: 862-8.

Rothwell, J. 1994. Control of human voluntary movement, 2nd ed. London: Chapman \& Hall.

Vodovnik, L., Rebersek, S., Stefanovaska, A., Zidar, J. 1988. Electrical stimulation for the control of paralysis and therapy of abnormal movement. Scand. J. Rehabil. Med., 17: 917.

Wang, R.Y., Chan, R.C., Tsai, M.W. 2000. Effect of thoraco-lumbas electric sensory stimulation on knee extensor spasticity of persons who survived cerebrovascular accident (CVA). J. Rehabil. Res. Dev., 37(1): 73-9. 